\title{
PERIANAL DERMITIS IN A 3.5 YEAR OLD TODDLER: PERIANAL STREPTOCOCCAL DERMITIS OR SEXUAL ABUSE?
}

\author{
E. Tsekoura, M.D., Ph.D., A. Karali M.D.
}

Pediatric Department, Asklepieion General Hospital, Athens, Greece

\section{Background}

Sexual abuse is a problem with epidemic characteristics in our society with $15 \%$ of men and $25 \%$ of women in their adulthood confirming a sexual abuse episode in their childhood. Thus is crucial for physicians to be able to recognize a possible sexual abuse case in order to protect the child as well as other children from repetitive episodes. On the other hand several medical conditions can be mistaken as sexual abuse and lead innocents in the court, separating children from their families

\section{Case Presentation}

A 3.5 year old toddler presented to the A\&E department with extensive perianal dermatitis, pain, redness, itching and clear borders (Fig 1).

He was given the history of a male who did this to him when he was in his father's house during Christmas Holidays. From the family-social history parents were divorced. Father was a drug user with good relationship to his ex wife.

Following query sexual abuse protocol we took cultures from the lesion including sexually transmitted diseases.

With the possible clinical diagnosis of Group A streptococcal perianal dermititis he was started on oral Amoxicillin-clavulanate and improved within 24 hours. Cultures were positive for GAS,

On developmental evaluation he had hyperactivityimpulsivity disorder.

Nevertheless he was constantly giving the same story of this man who did this to him and therefore the case was referred to social workers for further sexual abuse investigation.

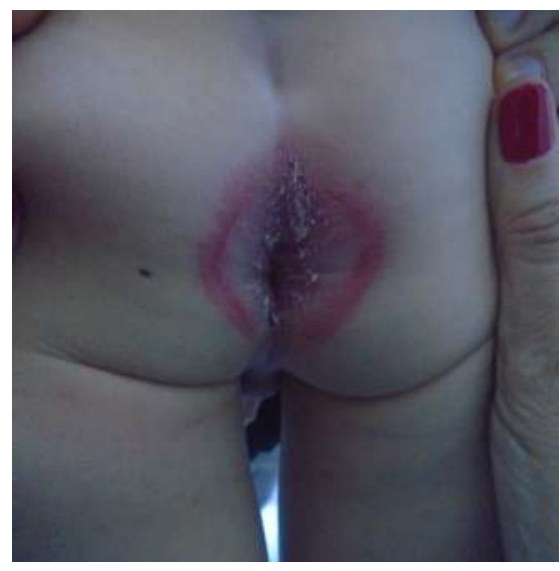

Fig 1

\section{Discussion}

GAS perianal dermatitis is commonly mistaken with sexual abuse as well as worm infection, fungal infection, constipation, and inflammatory bowel disease. It has a typical clinical presentation with clear borders perianal erythema, pain, itching and stool blood. Its prevalence is $1 / 1000$ pediatric $A \& E$ admissions. Despite typical presentation it is reported that $17 \%$ of doctors will suspect sexual abuse and $7 \%$ will investigate for inflammatory bowel disease.

Sexual abuse suspicion has always two dilemmas:

-If not identified will pose children under the risk of a repeated episode -Mistaken diagnosis will pose family and innocent people under a disastrous situation

In our case, despite the definite diagnosis of GAS perianal dermatitis the fact that the child presented the same story of sexual abuse legal authorities was informed for further investigation.

C.rig 1 